illuminated 'primaeval broth' at the dawn of life. Since they were possibly the first photosynthetic organisms, the whole of the spectrum not absorbed by water (from the edge of the ultraviolet to the near infrared) was available to them, which is probably why natural selection gave them the photo-synthetic pigment bacteriorhodopsin, with its broad absorption peak in the middle of this region.

Chlorophyll-based photosynthesis is much more complex. Bacteriorhodopsin uses light to transport protons across a membrane. Chlorophyll transports electrons instead. It too generates a proton gradient for ATP production, but does so indirectly as a result of relatively complex electron-processing reactions on either side of the photosynthetic membrane. To avoid the need to replicate these systems for each chlorophyll molecule, most of the pigment molecules are arranged in organized arrays around specialized molecules in reaction centres to which they pass their light energy by resonance transfer? ${ }^{3}$. Chlorophyll-based photosynthesis almost certainly evolved after bacteriorhodopsin-containing organisms were well established, when new photosynthetic organisms stood the best chance of survival if their photo-synthetic pigments did not absorb in the same region as bacteriorhodopsin. It is probably for this reason that nature selected the green pigment chlorophyll $a$, with its absorption peaks on either side of the rhodopsin peak.

The main advantage of chlorophyll is that its light-driven electron transport can drive redox systems, in particular the reduction of $\mathrm{CO}_{2}$. This ability almost certainly enabled the early chlorophyll-containing organisms to take over from those with bacteriorhodopsin as the primaeval broth became depleted of organic nutrients. With the near-extinction of the rhodopsin-containing bacteria, more light would have become available in the centre of the spectrum where chlorophyll $a$ does not absorb, allowing the evolution of a whole series of accessory pigments that absorb in this region and which pass on their energy to chlorophyll by resonance transfer.

Algae living in poor light in deep water have very effective accessory pigments. The combination of chlorophyll with phycoerythrin in the red algae covers a large part of the spectrum and the thallus of these organisms tends to be very dark in colour, approaching black, the 'ideal'. Similarly, the fucoxanthol of brown algae and diatoms complements chlorophyll very well. These pigments very efficiently pass their energy to the reaction centre ${ }^{3}$, but bear very little resemblance to chlorophyll, suggesting that they may have arrived by a long evolutionary pathway.

Where selection pressure was less intense, less drastic solutions are found.
Land plants, and the green algae living near the surface, are not short of light and a minor modification to some of their $b$ was sufficient. This brought the twin absorption peaks of the pigment closer towards the centre of the spectrum to absorb light missed by chlorophyll $a$, but there is still a gap in the middle where light can escape unabsorbed, giving these plants their characteristic green colour.

ANDREW GOLDSWORTHY

Department of Pure and Applied Biology,

Imperial College,

London SW7 $2 B B, U K$

1. Schreckenbach, T.H. in Phoyosynthesis in Relation to Model Systems (ed. Barber. J.) 189-209 (Elsevier, Amsterdam).

Calvin, M. Chemical Evolution (Clarendon, Oxford, 1969) 3. Hall, D.O. \& Rao, K.K. Photosynthesis (Edward Arnold London, 1987).

\section{Prospects of infrared prospecting for planets}

SiR-Interest in the search for planets around nearby stars has recently been fuelled by apparent evidence from the Infrared Astronomical Satellite (IRAS) of proto-planetary material orbiting $\mathrm{Vega}^{\prime}$ and possibly many nearby main-sequence stars $^{2}$. The discovery of what appears to be a disk viewed edge-on in $\beta$ Pictorus ${ }^{3}$ has given "Prospecting for planets in circumstellar dust" ${ }^{4}$ a further boost. While the presence of even a little dust around a solar-type star may be suggestive, unfortunately it makes any search for embedded planets a formidable task, at least in the infrared.

This can be illustrated by calculating what our Sun and its surrounding zodiacal dust look like in the infrared when viewed from 10 parsec $(\mathrm{pc})$ away in comparison to some of the Vega-like stars found by IRAS and to Earth- and Jupiter-size planets. Ten pc is taken as a representative distance known to contain about 50 solar-type stars. Good et al. ${ }^{5}$ have modelled the zodiacal cloud using IRAS data with a density

$r(R, T)=e r_{0}\left(\mathrm{R}_{0} / R\right)^{i . t} \exp \left[-4.2^{\circ}(Z / R)^{1.2}\right]$,

where $R_{0}=1 \mathrm{AU}, e r_{0}=1.1 \times 10^{-20} \mathrm{~cm}^{-1}$ between $R=0.1$ and 5 AU, zero elsewhere and the temperature distribution $T(R)=282\left(R_{0} / R\right)^{0.5}$. The table gives the calculated infrared flux from the Sun, the zodiacal dust, Jupiter and Earth, as seen from a distance of $10 \mathrm{pc}$. The Sun, Jupiter and Earth are taken to be $5,800 \mathrm{~K}, 135 \mathrm{~K}$ chlorophyll molecules to give chlorophyll

and $280 \mathrm{~K}$ black bodies, respectively. Results are given for 1 and 0.05 arc $\mathrm{s}$ beam diameters. A one arc $s$ diameter beam (equal to the diffraction limit for a $2-\mathrm{m}$ telescope at $12 \mu \mathrm{m}$ ) is just large enough to enclose the entire Solar System out to the orbit of Jupiter. With a 0.05 arc s beam, equivalent to $0.5 \mathrm{AU}$ at $10 \mathrm{pc}$ and requiring a 40-m telescope (array) in space, the telescope can be pointed as close as $1 \mathrm{Au}$ from the star without the warmest zodiacal dust or direct starlight entering the beam.

The table shows that the zodiacal flux in the one arc s diameter beam exceeds the flux from Jupiter by more than a factor of ten, but is only 0.002 of the photospheric flux from the Sun at $100 \mu \mathrm{m}$ and even less at 60,25 and $12 \mu \mathrm{m}$. Given that the uncertainty of photospheric flux models of solar-type stars is in the several per cent class, it is clear that evidence of zodiacal dust, not to mention any planets, would not be detectable in the IRAS data or with a 2-m class telescope. For nearby stars identified as Vega-like from IRAS data, the flux from the circumstellar disk exceeds the flux from the central star by factors 2.5-146 in the extreme case of $\beta$ $\mathrm{Pic}^{2}$. This is greater than three orders of magnitude more than the radiation due to zodiacal dust in our Solar System.

The flux from the zodiacal light with a 0.5-AU diameter beam pointed a distance of $1 \mathrm{AU}$ from the Sun beam would be about 60 per cent larger than the signal from an Earth-size planet. This ratio is encouraging. But the search for any embedded planets will be frustrated if the density distribution of the dust is not smooth and the diffracted light from the star is not almost totally suppressed.

I conclude that prospects for remote prospecting in the infrared for Earth and Jupiter-size planets in Vega-like circumstellar disks with any size telescope are virtually nil. Prospects are more encouraging for stars with dustclouds no larger than our zodiacal cloud. But that would require a telescope an order-ofmagnitude larger than will be built in the foreseeable future.

Hartmut H. Aumann

Jet Propulsion Laboratory,

California Institute of Technology,

Pasadena, California 91109, USA

\section{. Aumann, H.H. et al. Astrophys. J. 279, L23 (1984). Aumann, H. H. Publs astr. Soc. Pacil. 97,885 (1985). \\ Smith, B.A. \& Terrile, R.J. Science 226, 1421 (1984). \\ Diner, J.D. \& Appleby, J.F. Nature 322, 436 (1986). \\ Good, J.C., Hauser, M.G. \& Gautier, T.N. Advances in Space Research 1987, (in the press)}

Flux in the beam [Jy]

\begin{tabular}{clllll}
\hline \multirow{2}{*}{ micrometre } & \multicolumn{4}{c}{$\begin{array}{c}\text { 10-Au diameter beam } \\
\text { Sun centred }\end{array}$} & $\begin{array}{c}\text { 0.5-Au diameter beam } \\
\text { at 1AU from Sun }\end{array}$ \\
\cline { 2 - 5 } & Sun & Zodiacal & Jupiter & Earth & Zodiacal \\
12 & 1.73 & $1.3 \times 10^{-4}$ & $5.8 \times 10^{-7}$ & $4.6 \times 10^{-7}$ & $7.3 \times 10^{-7}$ \\
25 & 0.42 & $1.8 \times 10^{-4}$ & $6.4 \times 10^{-6}$ & $5.2 \times 10^{-7}$ & $8.5 \times 10^{-7}$ \\
60 & 0.07 & $1.0 \times 10^{-4}$ & $6.7 \times 10^{-6}$ & $1.9 \times 10^{-7}$ & $3.1 \times 10^{-7}$ \\
100 & 0.027 & $5.3 \times 10^{-5}$ & $3.7 \times 10^{-6}$ & $8.3 \times 10^{-8}$ & $1.4 \times 10^{-7}$ \\
\hline
\end{tabular}

